PROCEEDINGS OF THE

AMERICAN MATHEMATICAL SOCIETY

Volume 129, Number 12, Pages 3637-3645

S 0002-9939(01)06016-6

Article electronically published on April 25, 2001

\title{
INVERSE SCATTERING FOR THE NONLINEAR SCHRÖDINGER EQUATION II. RECONSTRUCTION OF THE POTENTIAL AND THE NONLINEARITY IN THE MULTIDIMENSIONAL CASE
}

\author{
RICARDO WEDER
}

(Communicated by Christopher D. Sogge)

\begin{abstract}
We solve the inverse scattering problem for the nonlinear Schrödinger equation on $\mathbf{R}^{n}, n \geq 3$ :

$$
i \frac{\partial}{\partial t} u(t, x)=-\Delta u(t, x)+V_{0}(x) u(t, x)+\sum_{j=1}^{\infty} V_{j}(x)|u|^{2\left(j_{0}+j\right)} u(t, x) .
$$

We prove that the small-amplitude limit of the scattering operator uniquely determines $V_{j}, j=0,1, \cdots$. Our proof gives a method for the reconstruction of the potentials $V_{j}, j=0,1, \cdots$. The results of this paper extend our previous results for the problem on the line.
\end{abstract}

\section{INTRODUCTION}

Let us consider the following nonlinear Schrödinger equation with a potential:

$$
i \frac{\partial}{\partial t} u(t, x)=-\Delta u(t, x)+V_{0}(x) u(t, x)+F(x, u), u(0, x)=\phi(x),
$$

where $t \in \mathbf{R}, x \in \mathbf{R}^{n}, n \geq 3$. The potential, $V_{0}$, is a real-valued function, $F(x, u)$ is a complex-valued function, and $\Delta:=\sum_{j=1}^{n} D_{j}^{2}$. We use the standard notation, $D_{j}:=\frac{\partial}{\partial x_{j}}$ and for $\alpha:=\left(\alpha_{1}, \cdots, \alpha_{n}\right), D^{\alpha}:=D_{1}^{\alpha_{1}} \cdots D_{n}^{\alpha_{n}}$, with $|\alpha|:=\sum_{j=1}^{n} \alpha_{j}$. We first construct the scattering operator for the nonlinear Schrödinger equation (1.1). For this purpose we introduce some assumptions and definitions.

Assumption A. Let $p$ satisfy $\rho<p<1+\frac{4}{n-2}$, where $\rho$ is the positive root of $\frac{n}{2} \frac{\rho-1}{\rho+1}=\frac{1}{\rho}$. Let $k$ be an integer such that $k>\frac{n}{p+1}$. Let $F=F_{1}+i F_{2}$ with $F_{1}, F_{2}$ real-valued, and $u=r_{1}+i r_{2}, r_{1}, r_{2} \in \mathbf{R}$. We suppose that $F(0)=0$ and that for all integers $\beta$ with $1 \leq \beta \leq k+1$ and all $\alpha$ with $\beta+|\alpha| \leq k+1$, we have that

$$
\sum_{j=1}^{2}\left|\frac{\partial^{\beta}}{\partial r_{1}^{\beta_{1}} \partial r_{2}^{\beta 2}} D^{\alpha} F_{j}(x, u)\right| \leq C|u|^{\max [0, p-\beta]} \text { for }|u| \leq \gamma,
$$

for some $\gamma>0$, and for all nonnegative integers, $\beta_{1}, \beta_{2}$, with $\beta=\beta_{1}+\beta_{2}$.

Received by the editors January 19, 2000 and, in revised form, April 27, 2000.

2000 Mathematics Subject Classification. Primary 35R30, 35Q55, 35P25, 81 U40.

This research was partially supported by Proyecto PAPIIT-DGAPA IN 105799.

The author is a Fellow of Sistema Nacional de Investigadores. 
We denote by $H_{0}$ the self-adjoint realization of $-\Delta$ in $L^{2}\left(\mathbf{R}^{n}\right)$ with domain the Sobolev space $W_{2,2}$. For the definition of the Sobolev spaces $W_{j, p}, j=1,2, \cdots, 1 \leq$ $p \leq \infty$, see [1].

Assumption B. We assume that $V_{0}$ is real valued and that for some $\delta>(3 n / 2)+1$,

$$
\sup _{x \in \mathbf{R}^{n}}(1+|x|)^{\delta}\left(\int_{|x-y| \leq 1}\left|D^{\alpha} V_{0}(y)\right|^{p_{0}} d y\right)^{1 / p_{0}}<\infty,
$$

for all $|\alpha| \leq k+k_{0}$, with $k$ as in Assumption A. If $n=3, p_{0}=2$ and $k_{0}=0$, and if $n \geq 4, p_{0}>n / 2$ and $k_{0}:=[(n-1) / 2]$, where $[\sigma]$ denotes the integral part of $\sigma$. Moreover, assume that zero is neither an eigenvalue nor half-bound state (a resonance) of $H:=H_{0}+V_{0}$.

Zero is said to be a half-bound state of $H$ if the equation $H \phi=0$ has a solution $\phi \notin L^{2}\left(\mathbf{R}^{n}\right)$, such that $(1+|x|)^{-1-\epsilon} \phi \in L^{2}\left(\mathbf{R}^{n}\right)$ for all $\epsilon>0$.

Under Assumption B $H$ is self-adjoint with domain $W_{2,2}$ and it has no singularcontinuous spectrum and no positive eigenvalues [5]. Moreover, the wave operators

$$
W_{ \pm}:=s-\lim _{t \rightarrow \pm \infty} e^{i t H} e^{-i t H_{0}}
$$

exist and Range $W_{ \pm}=\mathcal{H}_{c}$, the subspace of continuity of $H$. The scattering operator for the linear Schrödinger equation (equation (1.1) with $F=0$ ) is given by

$$
S_{L}:=W_{+}^{*} W_{-} .
$$

Actually, these results are true under more general conditions. The crucial issue is that Yajima has proven that under Assumption B the wave operators and the adjoints, $W_{ \pm}^{*}$, are bounded operators on $W_{l, p}, l=0,1, \cdots, k, 1 \leq p \leq \infty$. For this result see Theorem 1.2 of [25] and also [24]. This result and the intertwining relations for the wave operators, $e^{-i t H} P_{c}=W_{ \pm} e^{-i t H_{0}} W_{ \pm}^{*}$, imply that the following $L^{p}-L^{p}$ estimate follows from the corresponding result for $H_{0}$ (see [24]):

$$
\left\|e^{-i t H} P_{c}\right\|_{\mathcal{B}\left(L^{p}, L^{p}\right)} \leq \frac{C}{t^{n\left(\frac{1}{p}-\frac{1}{2}\right)}}, t>0,
$$

for $1 \leq p \leq 2$ and where $\frac{1}{p}+\frac{1}{p}=1$. By $P_{c}$ we denote the orthogonal projector onto $\mathcal{H}_{c}$. For any pair of Banach spaces $X, Y$, we denote by $\mathcal{B}(X, Y)$ the Banach space of all bounded operators from $X$ into $Y$. The $L^{p}-L^{p}$ estimate in $\mathbf{R}^{n}, n \geq 3$, was first proven, under slightly different conditions, in [8].

The results of Yajima 24] allow us to extend to the case of $n \geq 3$ the method for the construction of the scattering operator for (1.1) and for the solution of the inverse scattering problem that we gave in [23] in the case of $n=1$. The $L^{p}-L^{p}$ estimate and the continuity of the wave operators on $W_{k, p}$ for the problem on the line was proven in [20] and [21] (see also [6]).

Let us denote [13,

$$
N_{\delta}\left(V_{0}\right):=\sup _{x \in \mathbf{R}^{n}}\left[\int_{|x-y|<\delta}|V(y)|^{p_{0}} d y\right]^{1 / p_{0}} .
$$

Assumption C. We assume that $N_{\delta}\left(D^{\alpha} V_{0}\right)<\infty, \delta>0$, and that

$$
\lim _{\delta \rightarrow 0} N_{\delta}\left(D^{\alpha} V_{0}\right)=0
$$

where $|\alpha| \leq k-1$, with $k$ and $p_{0}$ as in Assumption B. 
We designate

$$
\begin{aligned}
M:= & \left\{u \in C\left(\mathbf{R}, W_{k, p+1}\right): \sup _{t \in \mathbf{R}}(1+|t|)^{d}\|u\|_{W_{k, p+1}}<\infty\right\}, \\
& \text { with norm : }\|u\|_{M}:=\sup _{t \in \mathbf{R}}(1+|t|)^{d}\|u\|_{W_{k, p+1}},
\end{aligned}
$$

where $d:=\frac{n}{2} \frac{p-1}{p+1}$. For functions $u(t, x)$ defined in $\mathbf{R}^{n+1}$ we denote $u(t)$ for $u(t, \cdot)$. In the following theorem we construct the small-amplitude scattering operator.

Theorem 1.1. Suppose that Assumptions A, B and C are satisfied and that $H$ has no eigenvalues. Then, there is a $\delta>0$ such that for all $\phi_{-} \in W_{k+1,2} \cap W_{k, 1+\frac{1}{p}}$ with $\left\|\phi_{-}\right\|_{W_{k+1,2}}+\left\|\phi_{-}\right\|_{W_{k, 1+\frac{1}{p}}} \leq \delta$ there is a unique solution, $u$, to (1.1) such that $u \in C\left(\mathbf{R}, W_{k, 2}\right) \cap M$ and

$$
\lim _{t \rightarrow-\infty}\left\|u(t)-e^{-i t H} \phi_{-}\right\|_{W_{k, 2}}=0 .
$$

Moreover, there is a unique $\phi_{+} \in W_{k, 2}$ such that

$$
\lim _{t \rightarrow \infty}\left\|u(t)-e^{-i t H} \phi_{+}\right\|_{W_{k, 2}}=0 .
$$

Furthermore, $e^{-i t H} \phi_{ \pm} \in M$ and

$$
\begin{gathered}
\left\|u-e^{-i t H} \phi_{ \pm}\right\|_{M} \leq C\left\|e^{-i t H} \phi_{ \pm}\right\|_{M}^{p}, \\
\left\|\phi_{+}-\phi_{-}\right\|_{W_{k, 2}} \leq C\left[\left\|\phi_{-}\right\|_{W_{k+1,2}}+\left\|\phi_{-}\right\|_{W_{k, 1+\frac{1}{p}}}\right]^{p} .
\end{gathered}
$$

The scattering operator $S_{V_{0}}: \phi_{-} \hookrightarrow \phi_{+}$is injective on $W_{k, 1+\frac{1}{p}} \cap W_{k+1,2}$.

Note that in Theorem 1.1 we do not restrict $F$ in such a way that energy is conserved. Moreover, for $n=3, \rho=2$ and $\lim _{n \rightarrow \infty} \rho=1$. We prove Theorem 1.1 in Section 2 extending to this case the proof given in [23] in the case of $n=1$. We construct the solution $u(t, x)$ by means of the contraction mapping theorem in a ball, $M_{R}$, of $M$ with small enough radius, $R$. It follows from Sobolev's imbedding theorem [1] that $|u(t, x)|<\gamma, t \in \mathbf{R}, x \in \mathbf{R}^{n}$, for all $u(t, x) \in M_{R}$. This is the reason why we only have to assume that (1.2) holds for $|u| \leq \gamma$. For results on scattering for the nonlinear Schrödinger equation in the case where $V_{0}=0$ see [16], [17], [18, [10, 9], [11, 3], 7], 2] and the references quoted there. In [8] the direct scattering for (1.1) with $F=F(u)$ was studied for $n \geq 3$. The corresponding inverse problem was considered in 19]. For the case of the nonlinear Klein-Gordon equation on the line see 22 .

Since we wish to reconstruct the potential, $V_{0}$, we consider the scattering operator that relates asymptotic states that are solutions to the linear Schrödinger equation with potential zero ((1.1) with $\left.V_{0}=F=0\right)$ :

$$
S:=W_{+}^{*} S_{V_{0}} W_{-} .
$$

The first step is to reconstruct $S_{L}$ from $S$.

Theorem 1.2. Suppose that the assumptions of Theorem 1.1 are satisfied. Then for every $\phi_{-} \in W_{k+1,2} \cap W_{k, 1+\frac{1}{p}}$,

$$
\left.\frac{d}{d \epsilon} S(\epsilon \phi)\right|_{\epsilon=0}=S_{L} \phi
$$


where the derivative in the left-hand side of (1.15) exists in the strong convergence in $W_{k, 2}$.

Corollary 1.3. Under the conditions of Theorem 1.1 the scattering operator, $S$, determines uniquely the potential $V_{0}$.

Proof. By Theorem 1.2S uniquely determines $S_{L}$. From $S_{L}$ we uniquely reconstruct the potential $V_{0}$ using the known results on the inverse scattering problem for the linear Schrödinger equation. See [4].

Let us now consider the case where $F(x, u)=\sum_{j=1}^{\infty} V_{j}(x)|u|^{2\left(j_{0}+j\right)} u$. As we prove below we can also reconstruct the $V_{j}, j=1,2, \cdots$.

Lemma 1.4. Suppose that the conditions of Theorem 1.1 are satisfied, and moreover, that $F(x, u)=\sum_{j=1}^{\infty} V_{j}(x)|u|^{2\left(j_{0}+j\right)} u$, for $|u| \leq \gamma$, for some $\gamma>0$, where $j_{0}$ is an integer such that $j_{0} \geq(p-3) / 2$, and where $V_{j} \in W_{k, \infty}$ with $\left\|V_{j}\right\|_{W_{k, \infty}} \leq$ $C^{j}, j=1,2, \cdots$, for some constant $C$. Then, for any $\phi \in W_{k+1,2} \cap W_{k, 1+\frac{1}{p}}$ there is an $\epsilon_{0}>0$ such that for all $0<\epsilon<\epsilon_{0}$ :

$i\left(\left(S_{V_{0}}-I\right)(\epsilon \phi), \phi\right)_{L^{2}}=\sum_{j=1}^{\infty} \epsilon^{2\left(j_{0}+j\right)+1}\left[\iint d t d x V_{j}(x)\left|e^{-i t H} \phi\right|^{2\left(j_{0}+j+1\right)}+Q_{j}\right]$,

where $Q_{1}=0$ and $Q_{j}, j>1$, depends only on $\phi$ and on $V_{l}$ with $l<j$. Moreover, for any $\dot{x} \in \mathbf{R}$, and any $\lambda \geq 1$, we denote $\phi_{\lambda}(x):=\phi(\lambda(x-\dot{x}))$. Then, if $\phi \neq 0$,

$$
V_{j}(x)=\frac{\lim _{\lambda \rightarrow \infty} \lambda^{n+2} \iint d t d x V_{j}(x)\left|e^{-i t H_{\lambda}} \phi_{\lambda}\right|^{2\left(j_{0}+j+1\right)}}{\iint d t d x\left|e^{-i t H_{0}} \phi\right|^{2\left(j_{0}+j+1\right)}} .
$$

Corollary 1.5. Under the conditions of Lemma 1.4 the scattering operator, $S$, determines uniquely the potentials $V_{j}, j=0,1, \cdots$.

Proof. By Corollary 1.3, $S$ uniquely determines $V_{0}$. Then the wave operators, $W_{ \pm}$, are uniquely determined, and by (1.14), $S$ uniquely determines $S_{V_{0}}$. Finally by (1.16) and (1.17) $S_{V_{0}}$ uniquely determines $V_{j}, j=1,2, \cdots$.

We reconstruct the potentials $V_{j}, j=0,1, \cdots$, in the following way. First we obtain $S_{L}$ from $S$ using (1.15). By the method in [4] for inverse scaterring for the linear Schrödinger equation we reconstruct $V_{0}$. We then reconstruct $S_{V_{0}}$ from $S$ using (1.14). Finally (1.16) and (1.17) give us, recursively, $V_{j}, j=1,2, \cdots$. Our formula (1.17) is an extension to our case of the reconstruction algorithm of [15]. In 15 Strauss proved that in the case $V_{0}=0$ and $F(x, u)=V(x)|u|^{p-1} u, x \in$ $\mathbf{R}^{n}, p>4$ if $n=1, p>3$ if $n=2, p \geq 3$ if $n \geq 3$, and $V(x)$ a real-valued potential whose derivatives up to order $l$ are bounded, with $l>3 n / 4$; then, the scattering operator uniquely determines $V$.

\section{ScAttering}

By Theorem 3 on page 135 of [14,

$$
\left\|\mathcal{F}^{-1}\left(1+q^{2}\right)^{k / 2}(\mathcal{F} f)(q)\right\|_{L^{p}}
$$

is a norm that is equivalent to the norm of $W_{k, p}, 1<p<\infty$. $\mathcal{F}$ denotes the Fourier transform. Then, by equation (1.2) of [24]

$$
\left\|(I+H)^{l / 2} f\right\|_{L^{p}}
$$


defines a norm that is equivalent to the norm of $W_{l, p}, l=0,1, \cdots, k, 1<p<\infty$. We will use this equivalence below without further comments. This implies that estimate (1.6) holds in the norm on $\mathcal{B}\left(W_{l, p}, W_{l, \dot{p}}\right), l=0,1, \cdots, k$.

The following inequality is proven in Theorem 9.2 on page 141 of [13]:

$$
\left\|\left(D^{\alpha} V_{0}\right) \phi\right\|_{L^{2}} \leq C_{1} N_{\delta}\left(D^{\alpha} V_{0}\right)\|\phi\|_{W_{2,2}}+C_{2} N_{1}\left(D^{\alpha} V_{0}\right)\|\phi\|_{L^{2}},
$$

where $C_{1}$ is independent of $\delta$. Let us denote $R(\rho):=(H+\rho)^{-1}$ and $R_{0}(\rho):=$ $\left(H_{0}+\rho\right)^{-1}$. Equation (2.3) implies that if Assumption $\mathrm{C}$ holds, given $a<1$, there is $\rho_{0}>0$ such that

$$
\left\|V_{0} R_{0}(\rho)\right\|_{\mathcal{B}\left(L^{2}\right)} \leq a<1
$$

for all $\rho \geq \rho_{0}>0$. Moreover, $\rho_{0}$ depends on $V_{0}$ only through $N_{\delta}\left(V_{0}\right)$. It follows that

$$
R(\rho)=R_{0}(\rho)\left(I+V_{0} R_{0}(\rho)\right)^{-1}=R_{0}(\rho) \sum_{l=0}^{\infty}(-1)^{l}\left(V_{0} R_{0}(\rho)\right)^{l}
$$

for all $\rho \geq \rho_{0}$. Taking derivatives in (2.5) term by term we prove that

$$
\|R(\rho)\|_{\mathcal{B}\left(W_{j, 2}, W_{j+2,2}\right)} \leq C, j=0,1,2, \cdots, k-1 .
$$

It follows that if $k$ is odd,

$$
\left\|\left(H_{0}+\rho\right)^{(k+1) / 2}(R(\rho))^{(k+1) / 2}\right\|_{\mathcal{B}\left(L^{2}\right)} \leq C .
$$

Then, for some constants $C_{1}, C_{2}$,

$$
C_{1}\|\phi\|_{W_{k+1,2}} \leq\left\|(I+H)^{(k+1) / 2} \phi\right\|_{L^{2}} \leq C_{2}\|\phi\|_{W_{k+1,2}} .
$$

In the case when $k$ is even we have that

$$
R(\rho)^{(k+1) / 2}=R(\rho)^{k / 2}(H+\rho)^{-1 / 2} .
$$

Again using Theorem 9.2 on page 141 of [13] we obtain that

$$
\left\|\left|V_{0}\right|^{1 / 2} \phi\right\| \leq C_{1}\left[N_{\delta}\left(V_{0}\right)\right]^{1 / 2}\|\phi\|_{W_{1,2}}+C_{2}\left[N_{1}\left(V_{0}\right)\right]^{1 / 2}\|\phi\|_{L^{2}} .
$$

Then, if $\rho$ is large enough,

$$
\left\|(H+\rho)^{1 / 2} \phi\right\|^{2}=\left(\left(H_{0}+V_{0}+\rho\right) \phi, \phi\right) \geq C\|\phi\|_{W_{1,2}}^{2},
$$

and we have that

$$
\left\|(H+\rho)^{-1 / 2}\right\|_{\mathcal{B}\left(L^{2}, W_{1,2}\right)} \leq C .
$$

Hence, by (2.6), (2.9) and (2.12), equation (2.8) also holds for $k$ even.

The proofs of Theorem 1.1, Theorem 1.2, and Lemma 1.4 follow as in 23]. We give details below for the convenience of the reader.

Proof of Theorem 1.1. By Sobolev's imbedding theorem [1] $L^{\infty}$ is continuously imbedded in $W_{k, 1+p}$ and it follows by standard arguments (see [9] and (2.16) below) that $u \in C\left(\mathbf{R}, W_{k, 2}\right) \cap M$ is a solution to (1.1) with $\lim _{t \rightarrow-\infty}\left\|u(t)-e^{-i t H} \phi\right\|_{W_{k, 2}}=0$ for some $\phi \in W_{k, 2}$, if and only if $u$ is a solution to the following integral equation:

$$
u(t)=e^{-i t H} \phi+\frac{1}{i} \int_{-\infty}^{t} e^{-i(t-\tau) H} F(x, u(\tau)) d \tau .
$$


Let us designate

$$
\mathcal{Q} u(t):=\frac{1}{i} \int_{-\infty}^{t} e^{-i(t-\tau) H} F(x, u(\tau)) d \tau .
$$

For $R>0$ let us denote $M_{R}:=\left\{u \in M:\|u\|_{M} \leq R\right\}$. By Assumption A, (1.6) and since $L_{\infty} \subset W_{k, p+1}$, there is an $R_{0}>0$ such that if $u \in M_{R_{0}}$,

$$
\|\mathcal{Q} u(t)-\mathcal{Q} v(t)\|_{W_{k, p+1}} \leq C(1+|t|)^{-d}\left(\|u\|_{M}+\|v\|_{M}\right)^{p-1}\|u-v\|_{M},
$$

where we used that $d>1$ and that $p d>1$. Moreover, by (2.15) with $v(t)=0$,

$$
\begin{aligned}
\|\mathcal{Q} u(t)\|_{W_{k, 2}}^{2} & \leq C \Re \int_{-\infty}^{t} d \tau\left((I+H)^{k / 2} F(x, u(\tau)),(I+H)^{k / 2} \mathcal{Q} u(\tau)\right)_{L^{2}} \\
& \leq C \int_{-\infty}^{t} d \tau\|F(x, u)(\tau)\|_{W_{k, 1+1 / p}} \times(1+|\tau|)^{-d}\|u\|_{M}^{p} \\
& \leq C \int_{-\infty}^{t} d \tau\|u\|_{W_{k, p+1}}^{p}(1+|\tau|)^{-d}\|u\|_{M}^{p} \\
& \leq C \int_{-\infty}^{t} d \tau(1+|\tau|)^{-d(p+1)}\|u\|_{M}^{2 p} \\
& \leq C(1+\max [0,-t])^{-(d+d p-1)}\|u\|_{M}^{2 p} .
\end{aligned}
$$

Let us first prove the uniqueness. For $u, v$ any pair of solutions to (1.1) that satisfy (1.10) we have that

$$
u(t)-v(t)=\mathcal{Q} u(t)-\mathcal{Q} v(t) .
$$

Let us denote $u_{T}:=\chi_{(-\infty, T)}(t) u(t)$, where $\chi_{(-\infty, T)}(t)$ is the characteristic function of $(-\infty, T), T \in \mathbf{R} . v_{T}$ is similarly defined. It follows from (2.17) that

$$
\left\|u_{T}(t)-v_{T}(t)\right\|_{\tilde{M}}<1 / 2\left\|u_{T}(t)-v_{T}(t)\right\|_{\tilde{M}} \text { for some } T \text { negative enough, }
$$

where $\tilde{M}$ is defined as $M$, but with a slightly smaller $p$. Then, $u(t)=v(t)$ for $t \leq T$, and the standard uniqueness result implies that $u=v$. The uniqueness of $\phi_{+}$is obvious by the unitarity of $e^{-i t H}$ in $L^{2}$.

By Sobolev's imbedding theorem,

$$
\begin{aligned}
\left\|e^{-i t H} \phi_{-}\right\|_{W_{k, p+1}} & \leq C\left\|e^{-i t H} \phi_{-}\right\|_{W_{k+1,2}} \leq C\left\|(H+I)^{(k+1) / 2} e^{-i t H} \phi_{-}\right\|_{L^{2}} \\
& =C\left\|(H+I)^{(k+1) / 2} \phi_{-}\right\|_{L^{2}} \leq C\left\|\phi_{-}\right\|_{W_{k+1,2}} .
\end{aligned}
$$

By (1.6) and (2.19):

$$
\left\|e^{-i t H} \phi_{-}\right\|_{M} \leq C\left[\left\|\phi_{-}\right\|_{W_{k+1,2}}+\left\|\phi_{-}\right\|_{W_{k, 1+\frac{1}{p}}}\right] .
$$

Let us take $R \leq R_{0}$ so small that $C(2 R)^{p-1} \leq 1 / 2$, with $C$ as in (2.15), and $\delta>0$ such that $C \delta \leq R / 4$, with $C$ as in (2.20). Then, the map $u \hookrightarrow e^{-i t H} \phi_{-}+\mathcal{Q} u$ is a contraction from $M_{R}$ into $M_{R}$ for all $\phi_{-} \in W_{k+1,2} \cap W_{k, 1+\frac{1}{p}}$ with $\left\|\phi_{-}\right\|_{W_{k+1,2}}+$ 
INVERSE SCATTERING FOR THE NONLINEAR SCHRÖDINGER EQUATION II 3643 $\left\|\phi_{-}\right\|_{W_{k, 1+\frac{1}{p}}} \leq \delta$. The contraction mapping theorem implies that this map has a unique fixed point that is a solution to (2.13) in $M_{R}$. Moreover,

$$
\|u\|_{M} \leq\left\|e^{-i t H} \phi_{-}\right\|_{M}+\frac{1}{2}\|u\|_{M},
$$

and then

$$
\|u\|_{M} \leq C\left\|e^{-i t H} \phi_{-}\right\|_{M} .
$$

Equation (1.12) for $\phi_{-}$follows from (2.13), (2.15) with $v=0$ and (2.22). By (2.13) and (2.16) $u \in C\left(\mathbf{R}, W_{k, 2}\right)$ and (1.10) holds.

We now define

$$
\phi_{+}=\phi_{-}+\frac{1}{i} \int_{-\infty}^{\infty} e^{i \tau H} F(x, u(\tau)) d \tau .
$$

Estimating as in (2.16) we prove that $\phi_{+} \in W_{k, 2}$ and that

$$
\left\|\phi_{+}-\phi_{-}\right\|_{W_{k, 2}} \leq C\|u\|_{M}^{p} .
$$

Equation (1.13) follows from (2.20), (2.22) and (2.24). By (2.13) and (2.23)

$$
u(t)=e^{-i t H} \phi_{+}-\frac{1}{i} \int_{t}^{\infty} e^{-i(t-\tau) H} F(x, u(\tau)) d \tau .
$$

We prove (1.11) estimating as in (2.16). In a similar way we prove that

$$
\left\|\int_{t}^{\infty} e^{-i(t-\tau) H} F(x, u(\tau)) d \tau\right\|_{M} \leq C\|u\|_{M}^{p},
$$

and it follows that

$$
\|u\|_{M} \leq C\left\|e^{-i t H} \phi_{+}\right\|_{M} .
$$

Equation (1.12) for $\phi_{+}$follows from (2.25), (2.26) and (2.27). We prove that $S_{V_{0}}$ is injective as in the proof of uniqueness above.

Proof of Theorem 1.2. Since $S(0)=0$ and $W_{ \pm}$are bounded on $W_{k, 2}$, it is enough to prove that

$$
s-\lim _{\epsilon \downarrow 0} \frac{1}{\epsilon}\left(S_{V}(\epsilon \phi)-\epsilon \phi\right)=0 .
$$

By (2.20) and (2.22) with $\phi_{-}$replaced by $\epsilon \phi$ we have

$$
\|u\|_{M} \leq C \epsilon\left[\left\|\phi_{-}\right\|_{W_{k+1,2}}+\left\|\phi_{-}\right\|_{W_{k, 1+\frac{1}{p}}}\right] .
$$

To prove (2.28) we estimate the integral on the right-hand side of (2.23) as in (2.16), with the aid of (2.29).

Proof of Lemma 1.4. By the contraction mapping theorem,

$$
u(t)=e^{-i t H} \epsilon \phi+\sum_{n=1}^{\infty} \mathcal{Q}^{n} e^{-i t H} \epsilon \phi .
$$

Equation (1.16) follows from (2.23) and (2.30). By Sobolev's imbedding theorem [1], $W_{k+1,2} \subset L^{q}, 2 \leq q \leq \infty$. Then, estimating as in (2.19) we prove that 
$\left\|e^{-i t H} \phi\right\|_{L^{q}} \leq C_{q}\left\|e^{-i t H} \phi\right\|_{W_{k+1,2}} \leq C_{q}\|\phi\|_{W_{k+1,2}}, 2 \leq q \leq \infty$, and it follows from (1.6) that

$$
\iint d t d x\left|e^{-i t H} \phi\right|^{2\left(j_{0}+j+1\right)}<\infty, j=1,2, \cdots .
$$

For $\lambda \geq 1$ and $\dot{x} \in \mathbf{R}^{n}$ we denote by $H_{\lambda}$ the following self-adjoint operator in $L^{2}$ :

$$
H_{\lambda}:=H_{0}+V_{\lambda}(x), \text { where } V_{\lambda}(x)=\frac{1}{\lambda^{2}} V_{0}\left(\frac{x}{\lambda}+\dot{x}\right) .
$$

Since $H$ has no eigenvalues, we have that $H_{\lambda}$ has no eigenvalues, i.e., $H_{\lambda}>0$. Moreover, as $N_{\delta}\left(D^{\alpha} V_{\lambda}\right) \leq N_{\delta}\left(D^{\alpha} V_{0}\right)$ for $\lambda \geq 1$, equation (2.8) holds with $H_{\lambda}$ instead of $H$ with the same $C_{1}, C_{2}$ for all $\lambda \geq 1$.

Let us denote $\tilde{t}:=\lambda^{2} t$ and $\tilde{x}:=\lambda(x-\dot{x})$. We have that

$$
\left(e^{-i \tilde{t} H_{\lambda}} \phi\right)(\tilde{x})=\left(e^{-i t H} \phi_{\lambda}\right)(x) .
$$

Equation (2.33) implies that

$$
\begin{aligned}
I_{j} & :=\lambda^{n+2} \iint d t d x V_{j}(x) \mid e^{-i t H_{\lambda}} \phi^{2\left(j_{0}+j+1\right)} \\
& =\iint d \tilde{t} d \tilde{x} V_{j}\left(\frac{\tilde{x}}{\lambda}+\dot{x}\right)\left|e^{-i \tilde{t} H_{\lambda}} \phi\right|^{2\left(j_{0}+j+1\right)}(\tilde{x}) .
\end{aligned}
$$

By Theorem VIII.20 on page 286 of [12] and (2.32)

$$
s-\lim _{\lambda \rightarrow \infty} e^{-i \tilde{t} H_{\lambda}} \phi=e^{-i \tilde{t} H_{0}} \phi,
$$

where the limit exists in the strong topology on $W_{k+1,2}$. By Sobolev's imbedding theorem, the limit in (2.35) also exists in the strong topology on $L^{q}, 2 \leq q \leq \infty$. Moreover,

$$
\left\|e^{-i \tilde{t} H_{\lambda}} \phi\right\|_{L^{\infty}} \leq C\|\phi\|_{W_{k+1,2}}
$$

By (1.6) and 2.33),

$$
\begin{aligned}
\left\|e^{-i \tilde{t} H_{\lambda}} \phi\right\|_{L^{p+1}}^{p+1} & =\lambda^{n}\left\|e^{-i t H} \phi_{\lambda}\right\|_{L^{p+1}}^{p+1} \leq C \frac{1}{t^{d(p+1)}} \lambda^{n}\left\|\phi_{\lambda}\right\|_{L^{1+1 / p}}^{p+1} \\
& =C \frac{1}{\tilde{t}^{d(p+1)}}\|\phi\|_{L^{1+1 / p}}^{p+1},
\end{aligned}
$$

with $d:=\frac{n}{2} \frac{p-1}{p+1}$. Equation (1.17) follows from (2.34), (2.35), (2.36), 2.37) and the dominated convergence theorem. Note that $2\left(j_{0}+j+1\right) \geq p+1$, that $d(p+1)>1$, and that $V_{j}$ is continuous.

\section{REFERENCES}

[1] R. A. Adams, "Sobolev Spaces", Academic Press, New York, 1975. MR 56:9247

[2] J. Bourgain, "Global Solutions of Nonlinear Schrödinger Equations", Colloquium Publications 46, A.M.S., Providence, 1999. MR 2000h:35147

[3] T. Cazenave, "An Introduction to Nonlinear Schrödinger Equations, Text. Met. Mat. 26, Univ. Fed. Rio de Janeiro, 1993.

[4] V. Enss and R. Weder, The geometrical approach to multidimensional inverse scattering, J. Math. Phys. 36 (1995), 3902-3921. MR 96f:81128

[5] R. Froese and I. Herbst, Exponential bounds and absence of positive eigenvalues for N-body Schrödinger operators, Commun. Math. Phys. 87 (1982/83) 429-447. MR 85g:35091 
[6] A. Galtbayar and K. Yajima, $L^{p}$ boundedness of wave operators for one-dimensional Schrödinger operators. Preprint 1999.

[7] J. Ginibre, "Introduction aux Équations de Schrödinger non Linéaires", Onze Édition, Paris, 1998.

[8] J. L. Journé, A. Soffer and C. D. Sogge, Decay estimates for Schrödinger operators, Comm. Pure Appl. Math. XLIV (1991), 573-604. MR 93d:35034

[9] T. Kato, Nonlinear Schrödinger equations, in "Schrödinger Operators", pp. 218-263, H. Holden and A. Jensen, eds., Lecture Notes in Physics 345, Springer-Verlag, Berlin, 1989. MR 91d:35202

[10] K. Mochizuki, On small data scattering with convolution nonlinearity, J. Math. Soc. Japan 41 (1989), 143-160. MR 90a:35207

[11] R. Racke, "Lectures in Nonlinear Evolution Equations. Initial Value Problems", Aspects of Mathematics E 19, F. Vieweg \& Son, Braunschweig/Wiesbaden, 1992.

[12] M. Reed and B. Simon, "Methods of Modern Mathematical Physics I: Functional Analysis", Academic Press, New York, 1972. MR 58:12429a

[13] M. Schechter, "Spectra of Partial Differential Operators, second edition", North Holland, Amsterdam, 1986. MR 88h:35085

[14] E. M. Stein, "Singular Integrals and Differentiability Properties of Functions", Princeton Univ. Press, Princeton, New Jersey, 1970.

[15] W. A. Strauss, Non linear scattering theory, in "Scattering Theory in Mathematical Physics", pp. 53-78, J.A. Lavita and J.-P. Marchand, editors, D. Reidel, Dordrecht-Holland / Boston, U.S.A.

[16] W. A. Strauss, Nonlinear scattering at low energy, J. Funct. Anal. 41 (1981) 110-133. MR 83b:47074a

[17] W. A. Strauss, Nonlinear scattering at low energy: sequel, J. Funct. Anal. 43 (1981) 281-293. MR 83b:47074b

[18] W. A. Strauss, "Nonlinear Wave Equations", CBMS-RCSM 73, American Mathematical Society, Providence, 1989. MR 91g:35002

[19] R. Weder, Inverse scattering for the nonlinear Schrödinger equation, Commun. Part. Diff. Equations 22 (1997), 2089-2103. MR 99c:35232

[20] R. Weder, $L^{p}-L^{p}$ estimates for the Schrödinger equation on the line and inverse scattering for the nonlinear Schrödinger equation with a potential, J. Funct. Analysis 170 (2000) 37-68. CMP 2000:07

[21] R. Weder, The $W_{k, p}$-continuity of the Schrödinger wave operators on the line, Comm. Math. Phys., 208 (1999), 507-520. CMP 2000:06

[22] R. Weder, Inverse scattering on the Line for the nonlinear Klein-Gordon equation with a potential, J. Math. Anal. and Appl., 252 (2000), 102-133. CMP 2001:05

[23] R. Weder, Inverse scattering for the nonlinear Schrödinger equation. Reconstruction of the potential and the nonlinearity. Preprint, 1999. To appear in Math. Meth. Appl. Sciences.

[24] K. Yajima, The $W^{k, p}$-continuity of the wave operators for Schrödinger operators, J. Math. Soc. Japan 47 (1995), 552-581. MR 97f:47049

[25] K. Yajima, The $W^{k, p}$-continuity of the wave operators for Schrödinger operators III, even dimensional cases $m \geq 4$, J. Math. Sci. Univ. Tokyo 2 (1995), 311-346. MR 96m:81247

Instituto de Investigaciones en Matemáticas Aplicadas y en Sistemas, Universidad Nacional Autónoma de México, Apartado Postal 20-726, México D.F. 01000

E-mail address: weder@servidor.unam.mx 